Eur Neurol 2003;49:180-182

DOI: 10.1159/000069072

\title{
Hepatitis-C-Associated Multiple Arterial Dissection of Cerebral Vessels
}

G.F. Hamann a, R. Zachoval b, H. von Einsiedel c, D. Sander ${ }^{d}$ Departments of a Neurology and ${ }^{b}$ Internal Medicine II, Klinikum Grosshadern, Ludwig Maximilian University, and Departments of ${ }^{c}$ Radiology and ${ }^{d}$ Neurology, Technical University, Munich, Germany

Neurological complications in hepatitis are common, and in most cases a polyneuropathy is described [1]. Cerebrovascular complications are rare but mostly seen in association with a systemic vasculitis or a cryoglobulinemia [2-4]. We describe an unusual case of cerebrovascular findings associated with hepatitis $\mathrm{C}$.

\section{Case Report}

A 48-year-old woman was admitted with right facial pain and an MRI suggestive of a right internal carotid artery (ICA) dissection. No further neurological deficit was noted but a right Horner syndrome. The patient had no vascular risk factors besides hypercholesterinemia. The family history did not evidence any stroke. Next day, she noticed left neck pain. Ultrasound revealed new dissections of the 

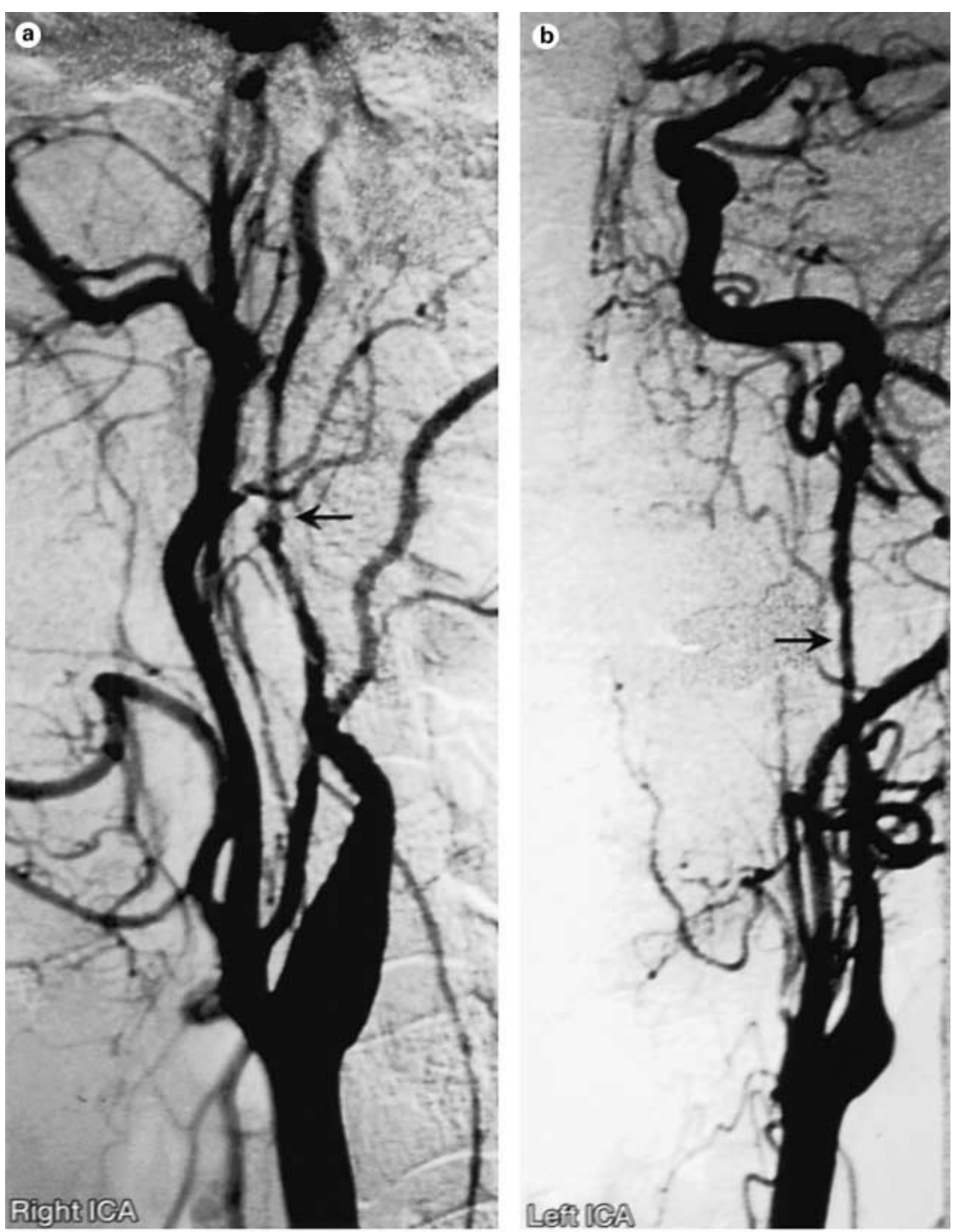

Fig. 1. a, b Angiography of the right and left ICA showing the dissection on both sides (black arrows). c Color-coded duplex sonography of the right vertebral artery, showing dissection (white arrow). d MRI ( $\mathrm{T}_{1}$-weighted, fat suppressed) showing the dissection of the right vertebral artery (white arrow) and semilunar hematoma of the vessel wall. e MRI ( $T_{1}$-weighted, fat suppressed) showing the dissections of both internal arteries (black arrows) and semilunar hematomas of the vessel walls.
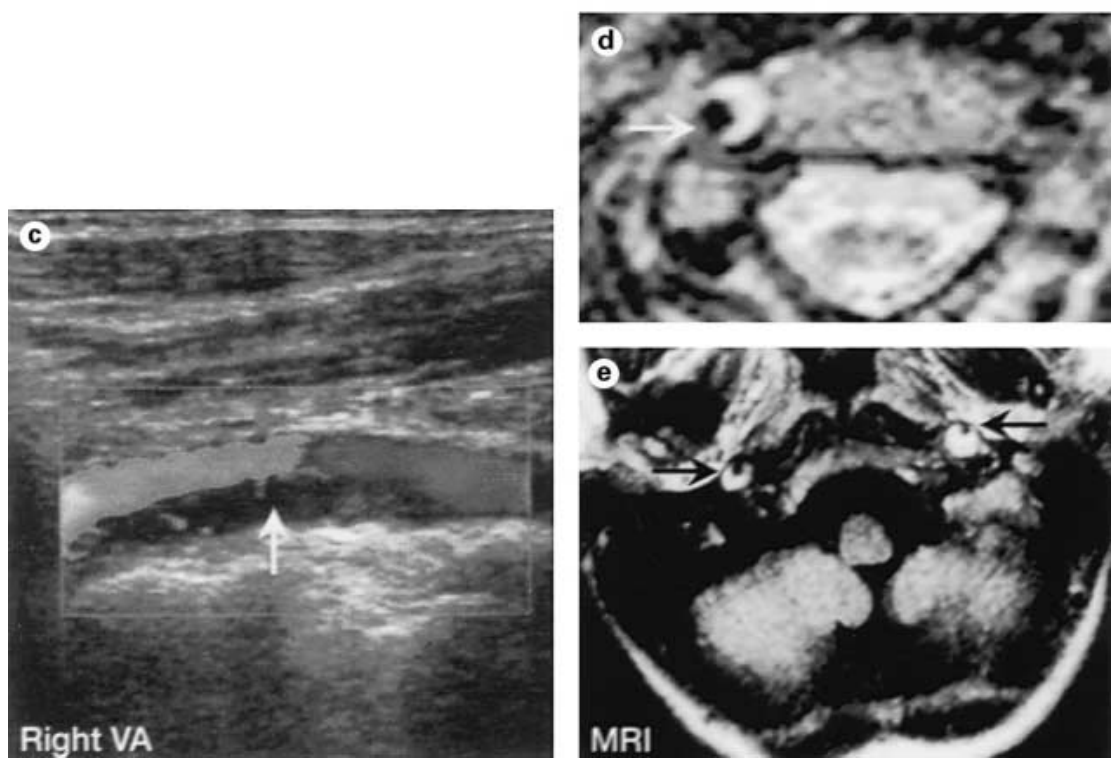

Short Reports 
left ICA and the right vertebral artery. Angiography proved the arterial dissections in the three major arteries supplying the brain (fig. 1). No signs of vasculitis, fibromuscular dysplasia or other vessel wall diseases were found; there was no history of neck manipulation or trauma. Possible causes like rheumatic, connective tissue, coagulation, inflammatory and autoimmune disorders were ruled out. Two weeks later, an axonal polyneuropathy (distal, symmetrical) was found and confirmed by nerve conduction studies and electromyography. Although she had never had an icterus nor a blood transfusion and no clinical evidence of hepatitis, anti-HCV antibodies were positive (ELISA). HCV RNA revealed 130,000 IU/ml; the HCV genotype was $2 \mathrm{a}$ (Simmonds). The transaminases were elevated (ALT 40 U/l, AST $27 \mathrm{U} / \mathrm{l})$. Cryoglobulins were elevated with $10 \mathrm{~g} / \mathrm{l}$ in serum. The liver biopsy showed lymphocytic, follicular infiltration but no necrosis, cirrhosis or cholestasis. The patient was put on interferon $\alpha_{2 b}, 3 \mathrm{MU}$ s.c. thrice weekly, and ribaverin, $800 \mathrm{mg} / \mathrm{day}$, for 6 months. ALT and AST rapidly normalized and HCV RNA became negative. Six months later, these promising results still hold. The polyneuropathy stabilized, both clinically and by nerve conduction studies and electromyography. Using ultrasound after 18 months, all vessels were normal without signs of dissection or stenosis.

\section{Discussion}

We report the very first case of arterial dissections of brain-supplying vessels associated with hepatitis C. Previous reports on extrahepatic manifestations of hepatitis $\mathrm{C}$ [1] focus on glomerulonephritis, porphyria cutanea tarda, Sjögren's syndrome and cryoglobulinemia. The polyneuropathy is the most prominent neurological finding in hepatitis C (10-20\% of all patients). Involvement of the central nervous system is thought to be caused by cerebral vasculitis [2]. The combination of hepatitis $\mathrm{C}$ and cryoglobulinemia is responsible for various extrahepatic complications [1,2]. The hepatitis-B-associated polyarteritis nodosa is prone to cerebrovascular complications mainly by a central nervous system vasculitis and by dissections of the aorta $[1,3,4]$ with a vasculitis of the vasa vasorum. So far, only a case of coronary dissection associated with hepatitis $\mathrm{C}$ has been described [5]. Since in rare instances polyarteritis nodosa may occur in hepatitis C, a similar pathophysiology can be hypothesized. Unfortunately, we can only report the association between the observed multiple cervical artery dissections and the hepatitis $\mathrm{C}$. This association is not defined as a causal relationship. The link between the two findings is mainly given by the tight temporal relationship. Further support for the association might be seen in the complete recovery from the dissection following treatment of the hepatitis $\mathrm{C}$ and the lack of any further dissections since the hepatitis was under control. In accordance with previous findings, our patient had no clinical signs of brain infarction [6]. Patients with Horner syndrome and lack of artery occlusion are significantly less endangered to develop ischemic symptoms [6].

Two central issues emerge from our case: when developing ischemic symptoms, hepatitis $\mathrm{C}$ patients should be screened for arterial dissections, and patients with dissections should be tested for hepatitis $\mathrm{C}$ when no other apparent reasons can be found.

\section{References}

1 Willson RA: Extrahepatic manifestations of chronic viral hepatitis. Am J Gastroenterol 1997;92:4-17.

2 Heckmann JG, Kayser C, Heuss D, Manger B, Blum HE, Neundörfer B Neurological manifestations of chronic hepatitis C. J Neurol 1999;246: 486-491.
3 Iino T, Eguchi K, Sakai M, Ishijima M, Toriyama K: Polyarteriitis nodosa with aortic dissection: Necrotizing vasculitis of the vasa vasorum. J Rheumatol 1992;19:1632-1636.

4 Petty GW, Duffy J, Huston J: Cerebral ischemia in patients with hepatitis C virus infection and mixed cryoglobulinemia. Mayo Clin Proc 1996;71: 671-678.

5 Fernandez-Gutierrez B, Zamorano J, Battle E, Alfonso F, Conde A, Sanchez-Harguindey L, Jover JA: Coronary dissection associated with hepatitis C virus-related cryoglobulinaemia. Rheumatology 1999;38:1299-1301.

6 Baumgartner RW, Arnold M, Baumgartner I, Mosso M, Gonner F, Studer A, Schroth G, Schuknecht B, Sturzenegger M: Carotid dissection with and without ischemic events: Local symptoms and cerebral artery findings. Neurology 2001;57:827-832.

Prof. Gerhard Hamann, Department of Neurology Ludwig Maximilian University, Klinikum Grosshadern Marchioninistrasse 15, D-81377 München (Germany) Tel. +49 89709536 70, Fax +49 8970953677

E-Mail hamann@brain.nefo.med.uni-muenchen.de 